\title{
The impact of escitalopram on vagally mediated cardiovascular function to stress and the moderating effects of vigorous physical activity: a randomized controlled treatment study in healthy participants
}

\author{
Camilla S. Hanson 1, Tim Outhred ${ }^{1,2,3}$, Andre R. Brunoni ${ }^{4}$, Gin S. Malhi ${ }^{2,3}$ and Andrew H. Kemp ${ }^{1,2,3,4 *}$ \\ SCAN Research and Teaching Unit, School of Psychology, University of Sydney, Sydney, NSW, Australia \\ 2 Discipline of Psychiatry, Sydney Medical School, Northern Clinical School, University of Sydney, Sydney, NSW, Australia \\ ${ }^{3}$ CADE Clinic, Royal North Shore Hospital, St. Leonards, NSW, Australia \\ ${ }^{4}$ Center for Clinical and Epidemiologic Research, University Hospital, University of São Paulo, São Paulo, Brazil
}

Edited by:

Arto J. Hautala, Verve Research,

Finland

Reviewed by:

Arto J. Hautala, Verve Research,

Finland

Daniel Boullosa, Universidade

Católica de Brasília, Brazil

${ }^{*}$ Correspondence:

Andrew H. Kemp, Center for Clinical and Epidemiologic Research,

University Hospital, University of São Paulo, Av. Prof. Lineu Prestes 2565, CEP 05508-000 Sao Paulo, Brazil

e-mail: andrew.kemp@hu.usp.br: andrew.kemp@sydney.edu.au
Recent concerns over the impact of antidepressant medications, including the selective serotonin reuptake inhibitors (SSRIs), on cardiovascular function highlight the importance of research on the moderating effects of specific lifestyle factors such as physical activity. Studies in affective neuroscience have demonstrated robust acute effects of SSRIs, yet the impact of SSRIs on cardiovascular stress responses and the moderating effects of physical activity remain to be determined. This was the goal of the present study, which involved a double-blind, randomized, placebo-controlled, cross-over trial of a single-dose of escitalopram $(20 \mathrm{mg}$ ) in 44 healthy females; outcomes were heart rate (HR) and its variability. Participants engaging in at least $30 \mathrm{~min}$ of vigorous physical activity at least 3 times per week (regular exercisers) showed a more resilient cardiovascular stress response than irregular vigorous exercisers, a finding associated with a moderate effect size (Cohen's $d=0.48$ ). Escitalopram attenuated the cardiovascular stress response in irregular exercisers only (HR decreased: Cohen's $d=0.80$; HR variability increased: Cohen's $d=0.33$ ). HR during stress under escitalopram in the irregular exercisers was similar to that during stress under placebo in regular exercisers. These findings highlight that the effects of regular vigorous exercise during stress are comparable to the effects of an acute dose of escitalopram, highlighting the beneficial effects of this particular antidepressant in irregular exercisers. Given that antidepressant drugs alone do not seem to protect patients from cardiovascular disease (CVD), longitudinal studies are needed to evaluate the impact of exercise on cardiovascular stress responses in patients receiving long-term antidepressant treatment.

Keywords: selective serotonin reuptake inhibitors (SSRIs), escitalopram, exercise, physical activity, cardiovascular stress response, heart rate, heart rate variability, HRV

\section{INTRODUCTION}

Depression and cardiovascular disease (CVD) are leading burdens of disease and this burden is projected to worsen up to 2030 and beyond with ageing of the population and increasing prevalence of multi-morbidity (Mathers and Loncar, 2006; Langan et al., 2013). Critically, depression increases risk for the development of CVD 1.5-fold, while patients with CVD and depression have a 2- to 3-fold increased risk of future cardiac events compared to those cardiac patients who do not have depression (Rudisch and Nemeroff, 2003). Psychological stress plays a key role in the development of depression (Cohen et al., 2007), and increases risk of mortality from a number of causes including CVD, cancer and external causes over an 8 year followup period (Russ et al., 2012). While many biological factors, including the hypothalamic-pituitary-adrenal (HPA) axis dysfunction and altered inflammatory processes, contribute to the relationship between stress, psychiatric illness and CVD, vagally mediated cardiovascular function-indexed by increases in heart rate (HR) and decreases in heart rate variability (HRV) - may underlie a substantial part of this risk (Thayer et al., 2010a; Nemeroff and Goldschmidt-Clermont, 2012). Importantly, the inhibitory function of the vagus nerve regulates both the HPA axis and inflammatory processes (Tracey, 2002; Thayer and Sternberg, 2006; Huston and Tracey, 2010; Kemp and Quintana, 2013), leading to proposals that chronic impairment in vagal function is an early marker of future morbidity and mortality (Kemp et al., 2010; Thayer et al., 2010b; Åberg et al., 2013; Kemp and Quintana, 2013).

Acute psychological stress is associated with parasympathetic withdrawal, sympathetic activation, an increase in HR and reductions in its variability (Madden and Savard, 1995; Porges, 1995; Steptoe and Kivimäki, 2012), while chronic stress is associated with persistent cardiovascular stress responses, which may then contribute to psychiatric illness, physical ill-health and all-cause 
mortality (Thayer and Brosschot, 2005; Thayer et al., 2010b; Lemogne et al., 2011; Åberg et al., 2013; Kemp and Quintana, 2013). Epidemiological research has even demonstrated that an increased resting HR increases the risk of suicide by $19-37 \%$ over a follow-up period of 9 years even after accounting for covariates including depressed mood (Lemogne et al., 2011). In another study lower cardiovascular fitness determined using the cycle ergonometric test at 18 years of age predicts an increased risk of suicide attempt and death by suicide over a follow-up period of 42 years ( $\AA$ berg et al., 2013). As lower cardiovascular fitness is associated with increased HR and reduced HR variability (Rennie, 2003), these findings highlight an important role of vagally mediated cardiovascular function in regards to psychological wellbeing as well as physical health. Importantly, increased parasympathetic control is associated with positive emotions (Kok and Fredrickson, 2010), resilience (Kashdan and Rottenberg, 2010) and improved regulation over the HPA axis and inflammatory processes (Tracey, 2002; Thayer and Sternberg, 2006; Huston and Tracey, 2010; Kemp and Quintana, 2013), thereby contributing to psychological wellbeing and physical health. National guidelines (Australian Government Department of Health and Ageing: http://www.health.gov.au/internet/main/publishing.nsf/ content/health-pubhlth-strateg-phys-act-guidelines) distinguish between regular moderate-intensity physical activity and regular vigorous exercise, recommending at least $30 \mathrm{~min}$ of vigorous exercise on top of regular activity for health and fitness benefits.

The selective serotonin reuptake inhibitors (SSRIs) are a firstline treatment option (Kemp et al., 2008) for both mood and anxiety disorders and admixtures of the two, and are also used widely in bipolar depression (Malhi, 2012). SSRIS have important acute neurophysiological effects (Kemp and Nathan, 2004; Kemp et al., 2004) providing the foundation upon which subsequent therapeutic response is based (Harmer et al., 2009). Acute effects are often studied in healthy samples to avoid confounds of illness and psychopathology (Harmer, 2010). Interestingly, acute SSRI treatment has been shown to attenuate cardiovascular responses to stress (Straneva-Meuse, 2004a; Golding et al., 2005; Jiang et al., 2013), However, longitudinal research indicates that they may also have adverse cardiovascular effects, including reductions in vagally mediated cardiovascular function (Licht et al., 2010b) and sudden cardiac death (Whang et al., 2009), highlighting the importance of research on antidepressant actions and the moderating effects of specific lifestyle factors such as physical activity. Physical activity is associated with improved psychological health (Powell et al., 2011; Åberg et al., 2013) and lower cardiovascular (and proinflammatory) responses to stress (Hamer and Steptoe, 2007) lowering the risk of future CVD (Mora et al., 2007; Hamer and Stamatakis, 2009; Hamer et al., 2009) and mortality (Leitzmann et al., 2007). The higher the parasympathetic activity at rest-indicated by lower HR and higher HRV- the more autonomic resources available to tackle subsequent stressors; a proposal known as the "autonomic resource hypothesis" (Hynynen et al., 2008; Boullosa et al., 2012). Thus, research on the impact of antidepressants and the moderating effects of physical activity has important implications for not only better understanding therapeutic response to antidepressant medications, but also for the long-term health of the cardiovascular system. Recent research in outpatients with coronary heart disease and depression (Blumenthal et al., 2012) demonstrates that exercise and sertraline (a SSRI) are equally effective at reducing depressive symptoms after 16 weeks of treatment, but that exercise led to greater improvements in HRV collected using 24-h Holter recordings compared with sertraline. However, it remains unclear, to what extent physical activity and SSRIs interact in moderating the effects of SSRIs on psychological stress.

Here we report on a proof-of-concept, laboratory-based, experimental study to examine the acute effects of a commonly prescribed SSRI, escitalopram, on HR and its variability under stress, with the aim of determining whether individual differences in levels of physical activity moderate these effects. Better understanding the impact of the SSRIs and the moderating effects that specific lifestyle factors exert has important implications for therapeutic responsiveness, psychological wellbeing, and physical health. We focus specifically on acute treatment effects in healthy female volunteers. We hypothesized that acute administration of escitalopram would attenuate increases in HR and decreases in HRV during stress, relative to placebo, and that regular vigorous exercise would facilitate this effect.

\section{METHODS}

\section{PARTICIPANTS}

Forty-four healthy female volunteers (aged 18-47, $M=23.70$, $S D=5.89)$ completed the study, and gave written informed consent in accordance with National Health Medical Research Council guidelines. Participants were recruited using universitywide staff and student newsletters. Participants were free from medication (other than hormonal contraceptives), physical and psychiatric illness, symptoms of depression and anxiety [PHQ-9 (Kroenke et al., 2001) and GAD-7 (Spitzer et al., 2006) assessment], illicit drug use, alcoholism, smoking, brain injury, neurological disorders, and sustained loss of consciousness. Only female participants were recruited for this study as females display higher rates of mental disorders (Nolen-Hoeksema, 2001; Kessler, 2003; Slade et al., 2009); focusing on females also allowed us to avoid known gender differences in baseline HRV (Rajendra Acharya et al., 2006), HRV responses to cognitive stress (Li et al., 2009), and responsiveness to SSRI treatment (Khan et al., 2005). Finally, participants abstained from caffeine on the morning of the experiment and no participant tested positive on pregnancy tests, which were conducted at each session. Ethics approval for the trial was secured from the University of Sydney's Human Research Ethics Committee (ref. 13901) and the Northern Sydney Central Coast Area Health Service Human Research Ethics Committee (ref. 1105-178 M), and it was registered with the Australian New Zealand Clinical Trials Registry (ANZCTR; ACTRN126111000719932).

\section{EXPERIMENTAL DESIGN}

Participants were randomly assigned to receive escitalopram (20 mg) or placebo per os, tested in a double-blind crossover design, with two sessions per participant separated by at least 1 week to ensure a sufficient drug washout of at least 5 half-lives $\left(t_{1 / 2}=26.7 \mathrm{~h}\right.$; Sogaard, 2005). Forty-five percent of participants received placebo at the first session. Relative to other commonly 
prescribed SSRIs, escitalopram has improved efficacy, a lower side effect profile, and greater selectivity for serotonergic transport proteins (Fernandez et al., 2007; Cipriani et al., 2009a). The maximum recommended dosage of $20 \mathrm{mg}$ was used to maximize receptor occupancy (Kasper et al., 2009) and subsequent physiological effects from a single dose.

\section{MEASUREMENT OF PHYSICAL ACTIVITY}

Participants reported the frequency in which they engaged in vigorous physical activity using the International Physical Activity Questionnaire (IPAQ; Craig et al., 2003), a widely used, reliable and valid self-report assessment. Physical activity data is transformed into energy expenditure estimates of metabolic equivalent tasks (MET), such that one MET is equivalent to the energy cost of sitting quietly for an hour (1 kcal/kg/h). Participants were categorized into low or high vigorous activity groups-according to national guidelines (Australian Government Department of Health and Ageing: http://www.health.gov.au/internet/main/publishing.nsf/content/ health-pubhlth-strateg-phys-act-guidelines). These guidelines recommend at least $30 \mathrm{~min}$ of vigorous physical activity 3 days a week on top of regular moderate-intensity activity for health and fitness benefits. Participants categorized in the low activity grouping did not meet the criteria of at least $30 \mathrm{~min}$ of vigorous physical activity 3 days a week, while participants in the high activity grouping met or exceeded this criterion. Groups did not differ on time spent sitting, walking or performing moderate physical activity (all $p>0.1$ ). Groups were compared on resting state $\mathrm{HR}$, providing a (partial) validation of questionnaire-based categorization (see participant characteristics).

\section{STRESS MANIPULATION}

The mental arithmetic task component of the Trier Social Stress Test (TSST; Kirschbaum et al., 1993) was used to elicit stress (Jönsson et al., 2010) and associated physiological correlates, including $\mathrm{HR}$, blood pressure, catecholamines and cortisol (Straneva-Meuse, 2004a; Vermetten, 2006). Participants were instructed to count backwards subtracting thirteens, beginning at either 2083 or 2027 for 5-min. Participants were given one of these two alternate versions of the arithmetic task across sessions. No feedback was given for correct responses, and the experimenter vocalized the word "error" when a mistake was made, instructing the participant to start counting from the beginning, further increasing stress and social pressure as per prior studies (Hjortskov et al., 2004).

\section{PROCEDURE}

Testing was conducted in a psychophysiology laboratory at the Clinical Assessment and Diagnostic Evaluation (CADE) Clinic (www.cadeclinic.com), Royal North Shore Hospital. Participants arrived in the early morning, having consumed breakfast, and abstained from caffeine, as caffeine increases sympathetic nervous system (SNS) activity (Sondermeijer et al., 2002). Participants completed a consent form and pregnancy test. Height and weight were measured at the first session to calculate BMI. Testing commenced $3 \mathrm{~h}$ after administration of either placebo or $20 \mathrm{mg}$ of escitalopram, so as to coincide with the time of the expected peak plasma concentration $\left(T_{\max }=3.0 \pm 1.5 \mathrm{~h}\right.$; Sogaard, 2005; Rao, 2007) During the waiting period, the participant was provided with a standardized snack and in the first session only, completed the IPAQ. The snack helped to minimize autonomic and mood changes associated with hunger. In order to determine the presence of side effects, subjective wellness was recorded during the waiting period and after psychophysiological testing. Participants reported whether they experienced side effects and were asked to guess their treatment condition in order to examine the potential impact of treatment unblinding at each session.

All participants were tested in the late morning, and at the same time on each session to control for potential changes in HRV with circadian rhythms (Kleiger et al., 2005). Participants sat in a reclined chair with their legs raised and were instructed to breathe normally, remain still and awake for 5-min of data collection in the resting state. Following this, 5-min recordings were made during the stress condition in which participants were required to perform the mental arithmetic task. The experimenter was seated in close proximity to the participant and acted in a formal manner, in order to further increase the stressful nature of the procedure through social pressure (Hjortskov et al., 2004). After testing, subjective ratings of relaxation and stress for each condition were recorded on a five-point Likert scale (from low to high) to determine the efficacy of the experimental manipulations.

Participants provided saliva samples $(1 \mathrm{~mL})$ for estradiol and progesterone analysis before treatment administration at each session. The hormonal saliva samples were stored frozen until assay. On the day of assay, samples were thawed for determination of salivary progesterone and estradiol using commercially available kits (Salimetrics, USA) according to the manufacturers instructions. Thawed samples were centrifuged at $1500 \times g$ for $15 \mathrm{~min}$ to collect clear saliva which was used without further processing for all assays. All samples were brought to room temperature before adding to assay wells and all samples were analyzed in duplicate. Hormonal menstrual phase was determined in accordance with previous research (Lu et al., 1999; Gandara et al., 2007).

\section{DATA COLLECTION AND ANALYSIS}

$\mathrm{HR}$ and R-R interval recordings - on which analyses of HRV were conducted-were made during two conditions, consistent with Task Force guidelines (Task Force of the European Society of Cardiology and the North American Society of Pacing and Electrophysiology, 1996): (1) a 5-min resting state and (2) a 5-min social stress task, using a Polar RS800CX training device at a sampling rate of $1000 \mathrm{~Hz}$. These devices have been validated against the electrocardiogram (ECG) and have excellent reliability, especially in young, healthy individuals in a supine position and when analysis is conducted on normalized values (Gamelin et al., 2006; Weippert et al., 2010) (see also Quintana et al., 2012), as was the case in the current study. This device is wirelessly connected to an electrode strap worn just beneath the chest, that has been wetted with saline solution $(0.9 \% \mathrm{NaCl})$ to simulate sweat and ensure conductivity. R-R intervals were extracted from text files and analyzed in Kubios HRV analysis software (version 2.0, 2008, biosignal analysis and medical imaging group, University of Kuopio, Finland, MATLAB). Each file was visually inspected for 
artifacts (ectopic and missing beats), and medium automatic filter corrections were applied to each data set. Measures of HRV included HF HRV (normalized units, n.u.) $(0.15-0.40 \mathrm{~Hz})$ and the Root Mean Square of the Successive Differences (RMSSD).

\section{STATISTICAL ANALYSIS}

All statistical analyses were conducted using IBM SPSS Statistics (Version 20) for Windows 7. The impact of escitalopram and the moderating effects of physical activity levels on $\mathrm{HR}$ and heart rate variability (HRV) were examined using mixed withinand between-subjects analysis of variance (ANOVA). Within subject factors were treatment (escitalopram vs. placebo) and task (stress vs. rest) and the between subjects factor was physical activity (high vs. low). A factor of "treatment order" was not included in statistical analysis for parsimony; order effects are rare, are generally underpowered even when an appreciable effect is present, and sufficient drug washout ameliorates such concerns (Senn, 1994; Senn et al., 2004; Mills et al., 2009). Independent samples $t$-tests were conducted to determine whether low and high activity groupings differed across demographic variables (Table 1). ANOVA was also employed to determine whether subjective ratings of relaxation and stress differed across treatment sessions and physical activity groupings. Cohen's $d$ effect size statistics were calculated for each pairwise comparison. Cohen's guidelines (Cohen, 1988a,b) identify $0.2,0.5$, and 0.8 as small, medium, and large effects, respectively. Partial eta-squared $\left(\eta_{p}^{2}\right)$ was reported for ANOVA effects as an indicator of effect size ( large $=0.14$; (Cohen, 1988a,b). The statistical threshold of 0.05 (two-tailed) was set for all analyses, except where directional effects were expected, in which one-tailed tests were employed. Manipulation checks included a focus on whether hormone or menstrual status, or treatment blinding differentially impacted on the results reported below. With respects to hormone and menstrual status, $\chi^{2}$ tests were conducted to check for equal proportions of participant menstrual phase within treatment sessions and equal proportions of participants using hormonal contraceptives in each order of treatment. Regression analyses were also conducted on hormone concentrations in order to determine the relationships between hormone levels and the HR and HRV responses under each treatment and task condition. With respects to treatment blinding, $\chi^{2}$ tests were performed to examine the relationship between guessing treatment condition and experiencing side effects. The effect of guessing and side effects on HR and HRV under each treatment and task condition was determined using repeated-measures ANOVAs on HR and HRV separately.

\section{RESULTS}

\section{PARTICIPANT CHARACTERISTICS}

Participant characteristics are presented in Table $\mathbf{1}$ and flow of participants through the different stages of the study is presented in Figure 1. The two physical activity groupings were matched in terms of age, BMI, years of education, time spent sitting, walking or performing moderate physical activity (all $p>0.1$ ). The high activity group spent more minutes in the past week in vigorous activity $\left[t_{(38)}=6.93, p<0.001, d=2.26\right]$, and had a higher weekly energy expenditure $\left[t_{(38)}=3.50, p=0.001, d=\right.$ 1.12] relative to the low activity group. Regardless of treatment, resting HR was significantly lower in the high activity group in comparison to the low activity group $\left[t_{(38)}=1.71, p=0.048\right.$ (one-tailed), $d=0.55]$, suggesting cardiovascular adaptations in the high activity group that may be explained by the link between bradycardia, regular exercise and physical fitness. Four participants were identified as multivariate outliers on HR variables using Tabachnick and Fidell's (2007) multivariate outliers identification procedure, and were subsequently excluded from further analysis. Forty participants aged between 18 and $47(M=23.70$, $S D=5.89)$ were included in final analyses.

\section{HORMONAL RESULTS}

The $\chi^{2}$ tests demonstrated that participants were equally distributed between menstrual phases on placebo, $\chi_{(2)}^{2}=0.950$, $\mathrm{p}=0.622$, and escitalopram, $\chi_{(2)}^{2}=0.950, p=0.622$, treatment sessions. There was an equal distribution of participants who took hormonal contraceptives to those who did not, $\chi_{(2)}^{2}=0.100$, $p=0.752$ and hormonal contraceptive status was equally distributed between those who received placebo or escitalopram at their first session, $\chi_{(1)}^{2}=0.002, p=0.962$. Regression analyses on HR and HRV data for each task and treatment condition showed no significant associations with progesterone or estradiol concentration [all $p>0.05$ (Bonferroni corrected for multiple analyses)]. Additionally, there were no differences between those who did and did not take hormonal contraceptives on each task and treatment condition $\left[\mathrm{HR}, F_{(1,38)}=0.544, p=0.465\right.$; HRV, $\left.F_{(2,37)}=0.733, p=0.487\right]$.

\section{TREATMENT BLINDING MANIPULATION CHECK}

A greater proportion of participants (70\%) were found to correctly guess treatment condition than that which was expected by chance, $\chi_{(1)}^{2}=6.400, p=0.011$. Side effects also occurred in $48 \%$ of the sample, although these did not occur in a greater proportion than that expected by chance, $\chi_{(1)}^{2}=0.100, p=0.752$. There was, however, a significant association between the subjective experience of side effects and correct treatment guessing, $\chi_{(1)}^{2}=6.535, p=0.011$, indicating that participants who correctly guessed treatment condition were likely to have made this correct guess based on their experience of side effects. Repeatedmeasures ANOVAs testing the interaction between presence of side effects and correct guessing of treatment were performed on the HR and HF HRV measures. Importantly, no significant interaction effects of correct guessing $\times$ side effects $\times$ treatment $\times$ task on $\operatorname{HR}\left[F_{(1,36)}=0.428, p=0.517\right]$ or $\operatorname{HRV}\left[F_{(1,35)}=0.995\right.$, $p=0.380]$ were observed.

\section{SUBJECTIVE RATINGS OF TASK}

A significant interaction between task and physical activity groupings on subjective ratings was observed, $\left[F_{(1,32)}=7.17\right.$, $\left.p=0.012, \eta_{p}^{2}=0.18\right]$. Follow-up pairwise comparisons demonstrated that participants in the high physical activity group reported significantly lower mental stress during the stress task, than the low physical activity group, $t_{(32)}=2.84, p=0.008$, $\mathrm{d}=1.00$. Therefore, the less physically active group found the mental stress task more stressful than the more physically active 
Table 1 | Participant demographic information.

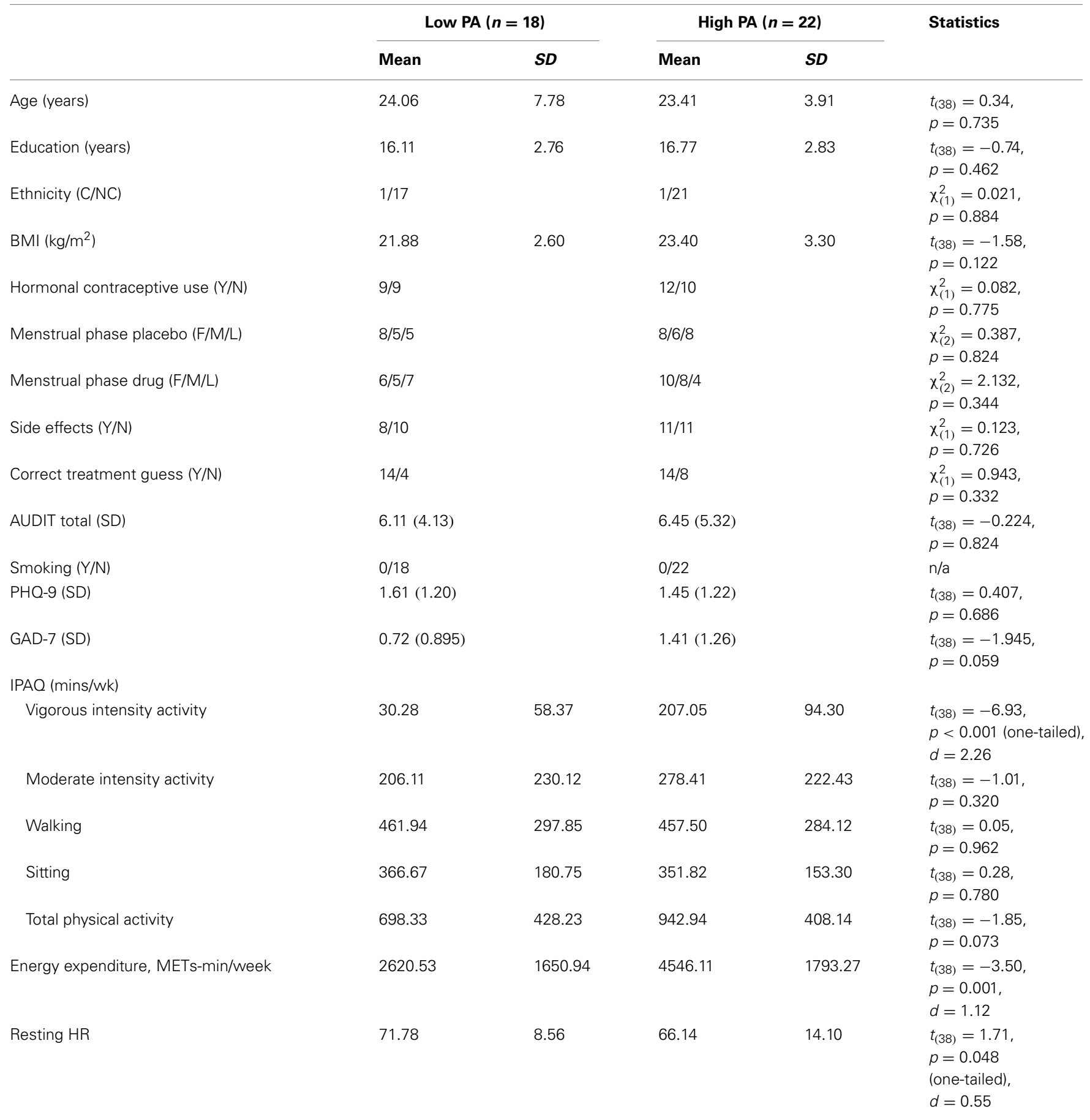

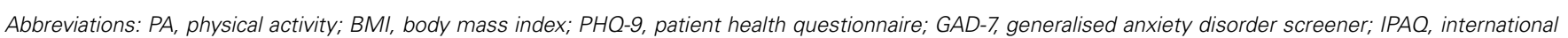
physical activity questionnaire; MET, metabolic equivalent task.

group, regardless of treatment. Importantly, however, a main effect of task was also observed indicating that participants were more stressed in the arithmetic task than during the rest condition $\left[F_{(1,32)}=102.77, p<0.001, \eta_{p}^{2}=0.76\right]$, confirming the validity of the stress condition in our sample, regardless of physical activity groupings. No main effects of physical activity $\left[F_{(1,32)}=\right.$ $2.49, p=0.124]$ or treatment $\left[F_{(1,32)}=2.35, p=0.135\right]$, or interactions between treatment and task $\left[F_{(1,32)}=2.26, p=\right.$ $0.142]$ or treatment, task, and physical activity $\left[F_{(1,32)}=0.228\right.$, $p=0.637]$ were observed on subjective ratings. 


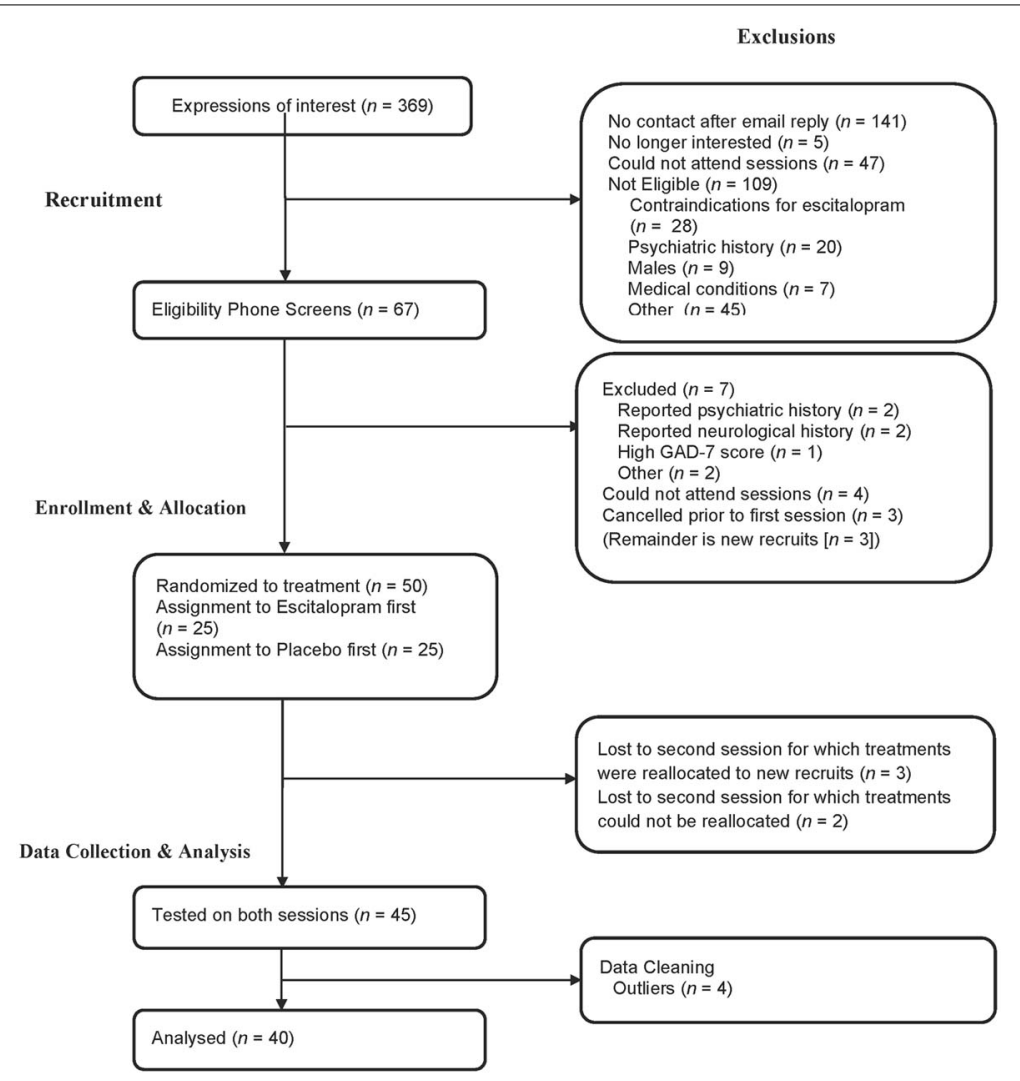

FIGURE 1 | A depiction of participants' attrition from the experiment and the reasons and stages at which they were excluded.

\section{IMPACT OF ESCITALOPRAM AND THE MODERATING EFFECTS OF PHYSICAL ACTIVITY}

Descriptive statistics for HR and HRV in high and low activity groupings for task and treatment are presented in Figure 2 and Table 2.

\section{Heart rate}

A three-way interaction between treatment, task and activity level was significant, $\left[F_{(1,38)}=7.18, p=0.011 \eta_{p}^{2}=0.16\right]$. Relative to placebo, escitalopram reduced $\mathrm{HR}$ in both the low activity $\left[t_{(38)}=2.80, p=0.008, d=0.45\right]$ and high activity $\left[t_{(38)}=\right.$ $2.24, p=0.031, d=0.36]$ groups during rest. Escitalopram also reduced $\mathrm{HR}$ in the low activity group $\left[t_{(38)}=4.97, p<0.001\right.$, $d=0.80]$ during stress. By contrast, escitalopram did not reduce $\mathrm{HR}$ in the high activity group during stress (Figure 2). Notably, HR for the high activity group during stress under placebo, was markedly reduced relative to those in the low activity group $\left[t_{(38)}=2.13, p=0.040, d=0.48\right]$, and equivalent to HR for the low activity group under drug (Figure 2), indicating that those in the high activity group display a more resilient cardiovascular response to stress than those in the low activity group. This interpretation is further supported by the observation of an interaction between task and physical activity grouping on subjective ratings of task (reported above). These findings were observed on a background of a significant treatment $\times$ task interaction $\left[F_{(1,38)}=6.61, p=0.014, \eta_{p}^{2}=0.15\right]$, a main effect of treatment
$\left[F_{(1,38)}=22.18, p<0.001, \eta_{p}^{2}=0.37\right]$, and a main effect of task $\left[F_{(1,38)}=210.43, p<0.001, \eta_{p}^{2}=0.85\right]$ collapsed across physical activity groupings. These findings indicate that the increase in HR under stress (relative to rest) was decreased by escitalopram (a treatment $\times$ task interaction), that HR was decreased by escitalopram (a main effect of treatment) and that HR was increased under stress (a main effect of task).

\section{Heart rate variability}

No significant three-way interaction was found for HRV $\left[F_{(2,37)}=0.23, p=0.80\right]$. Univariate tests for the three-way interaction between treatment, task and vigorous activity were not significant for $\operatorname{HF} F_{(1,38)}=0.06, p=0.804$, or RMSSD, $F_{(1,38)}=0.47, p=0.496$. However, a main effect of task was observed indicating that HRV was decreased by stress, relative to the rest, regardless of treatment [RMSSD, $F_{(1,38)}=35.99$, $p<0.001, d=0.96$; and HF, $F_{(1,38)}=84.60, p<0.001, d=$ 1.47]. Interestingly, post-hoc, pairwise comparisons indicated that escitalopram-relative to placebo-increased RMSSD (but not $\mathrm{HF})$ during stress in the low activity group $\left[t_{(37)}=2.07, p=\right.$ $0.046, d=0.33$ ], mirroring the findings of HR. No other pairwise comparisons were significant.

\section{DISCUSSION}

Recent reports on the adverse cardiovascular effects of the secondgeneration antidepressants (Whang et al., 2009; Licht et al., 

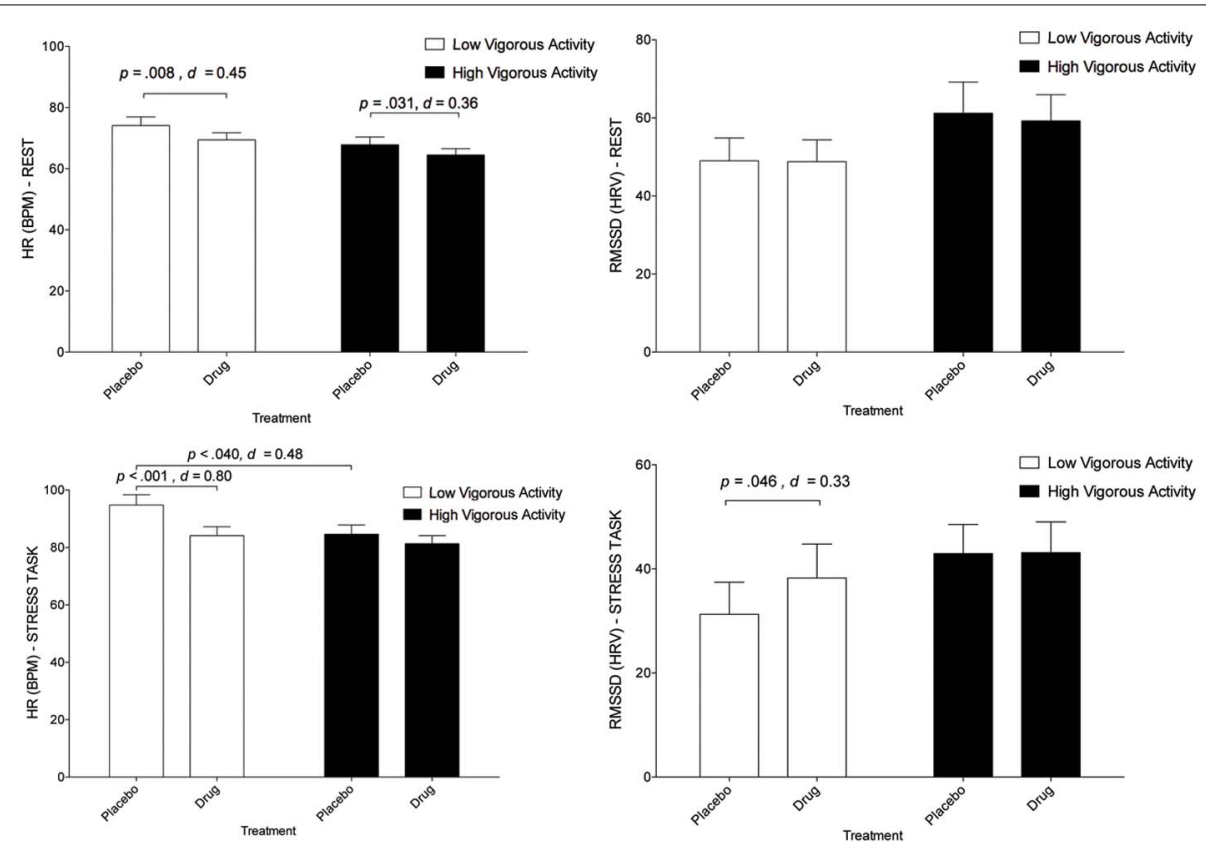

FIGURE 2 | The interaction between treatment and physical activity category on cardiovascular responses (with standard error bars). Top left graph: Effects on $H R$ at rest. Top right

graph: effects on RMSSD at rest. Lower left graph: Effects on HR during stress. Lower right graph: effects on RMSSD during stress.

Table 2 | HR and RMSSD means and standard deviations for vigorous exercise groups under each treatment for rest and stress conditions.

\begin{tabular}{|c|c|c|c|c|c|c|c|}
\hline & & \multicolumn{2}{|c|}{ Low vigorous activity } & $\% \Delta$ & \multicolumn{2}{|c|}{ High vigorous activity } & $\% \Delta$ \\
\hline & Stress & $94.80(10.45)$ & $84.12(10.03)$ & -11.2 & $84.62(17.93)$ & $81.34(15.21)$ & -3.9 \\
\hline & $\% \Delta$ & 27.9 & 21.2 & & 24.7 & 26.2 & \\
\hline & $\% \Delta$ & -36.2 & -21.6 & & -29.9 & -27.2 & \\
\hline
\end{tabular}

2010a), highlight the importance of research on specific lifestyle factors such as physical activity. Major findings observed here were that (a) participants in the high physical activity group (regular vigorous exercisers) reported feeling less stressed than those in the low physical activity grouping (irregular exercisers) during the stress task (i.e., an interaction between task and physical activity groupings on subjective ratings on task); this finding was associated with a large effect size (Cohen's $d=1.00$ ), (b) all participants reported feeling more stressed in the stress vs. rest condition (i.e., a main effect of task), a finding associated with a large effect size $\left(\eta_{p}^{2}=0.76\right)$, providing an important validation of our task in the context of an interaction between group and physical activity grouping, (c) irregular-relative to regular-exercisers display attenuated cardiovascular responses to stress following treatment with escitalopram (i.e., a three-way interaction between treatment, task and activity for HR, also indicated in a posthoc test on HRV), a finding associated with a moderate to large effect size (HR: Cohen's $d=0.80$; RMSSD HRV: Cohen's $d=$ 0.33 ), and (d) regular-relative to irregular-exercisers displayed a markedly lower HR under placebo (i.e., a three-way interaction between treatment, task and activity for HR), a finding associated with a moderate effect size (Cohen's $d=0.48$ ).

We show here that irregular exercisers-those participants that did not engage in $30 \mathrm{~min}$ of vigorous physical activity at least 3 days a week-reported feeling more stressed after the stress task and following an acute dose of escitalopram treatment, they displayed improvements in vagally mediated cardiovascular function during stress. Importantly, these subjective and objective findings relating to the impact of acutely administered escitalopram during stress were also observed regardless of physical activity grouping. The interactions with physical activity simply highlight that the beneficial effects of escitalopram were greatest in irregular exercisers. This effect was most prominent for HR, but was also observed in planned comparisons for HR variability. The 
impact of escitalopram on HRV under stress was not as robust as the observed findings for HR, however, this observation is understandable because under conditions of stress, parasympathetic activity, which is the primary driver of changes in HRV, is withdrawn and this reduces the capacity of other factors to moderate reductions in HRV during stress (observed as a main effect of task).

While animal and human studies (Babyak et al., 2000; Engesser-Cesar et al., 2007; Arunrut et al., 2009) have indicated that the combination of exercise and antidepressant medication may confer no advantage over either treatment alone, there is another issue at stake here. Although antidepressant medication-including the tricyclic medications, the serotonin and noradrenaline reuptake inhibitor and the selective serotonin reuptake inhibitors - may have short- to medium-term benefits including the amelioration of depressive symptoms and increased resilience to stress, research has begun to highlight the longerterm adverse cardiovascular effects of antidepressants (Whang et al., 2009; Licht et al., 2010a). Reductions in HRV have been attributable to the specific effects of antidepressants (Licht et al., 2008, 2010a) while increases in HRV are associated with their cessation (Licht et al., 2010a). An epidemiological study (Whang et al., 2009) on 63,469 women aged 30-55 years without baseline coronary heart disease $(\mathrm{CHD})$ reported that while depressive symptoms were associated with fatal $\mathrm{CHD}$, antidepressant use (61\% of participants were using an SSRI) was specifically associated with a 3.34 increased risk for sudden cardiac death even after controlling for a variety of confounds. A more recent study has reported that reduced cardiovascular fitness is associated with an increased risk of suicide over a 42 year follow-up period ( $\AA$ berg et al., 2013) highlighting the importance of cardiovascular health over the lifespan. Together, these findings highlight the importance of research on the cardiovascular effects of antidepressants and specific lifestyle factors such as physical activity. The present study makes an important contribution to this effort highlighting that the cardiovascular effects of regular vigorous exercise and of an acute dose of escitalopram during stress are comparable, i.e., both are associated with a decrease in HR and an increase in HRV.

Here we propose a simplified model for the beneficial effects of regular vigorous physical activity and SSRI treatment on vagal tone, initiating a path toward mental and physical wellbeing (see Figure 3). Increased vagal function is associated with increased psychophysiological flexibility (Thayer et al., 2009; Kashdan and Rottenberg, 2010) and stress resilience, which in turn promotes wellbeing (Oveis et al., 2009; Geisler et al., 2010, 2013; Kashdan and Rottenberg, 2010; Kok and Fredrickson, 2010; Thayer et al., 2010b; Kemp and Quintana, 2013). Increased vagal function also initiates downstream neurophysiological changes such as enhanced growth factors (Follesa et al., 2007), improved regulation of HPA axis function and inflammatory processes (Tracey, 2002; Thayer and Sternberg, 2006; Huston and Tracey, 2010; Pavlov and Tracey, 2012), leading to improved wellbeing (Thayer et al., 2010b; Kemp and Quintana, 2013). The model also highlights the direct links between regular vigorous physical activity and wellbeing (discussed above). However, it is noted that overtrained athletes also display a decease in HRV, which limits the autonomic resources available to respond to emotional and physical stress (Hynynen et al., 2008; Boullosa et al., 2012).

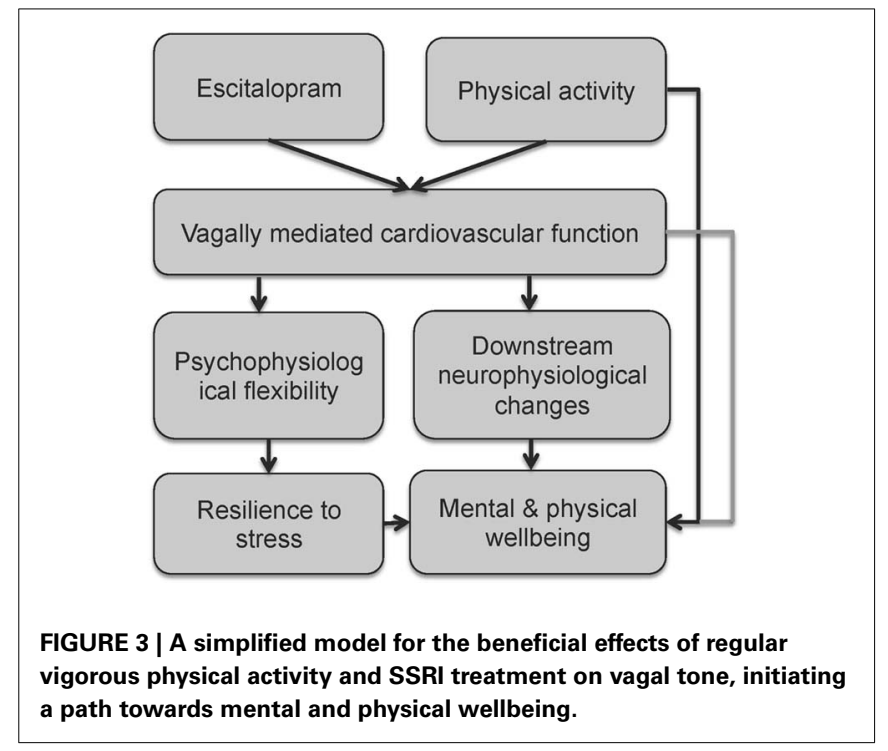

In the present study we demonstrated that acute administration of escitalopram is associated with increased vagal function under both resting and task conditions; a finding that was particularly robust for HR. This finding is consistent with reported short- to medium-term beneficial effects of SSRIs on cardiovascular and neuroendocrine responses to stress in depressed patients (e.g., Straneva-Meuse, 2004b), but contrast against longer-term research outcomes (Whang et al., 2009; Licht et al., 2010a) highlighting the adverse effects of the SSRI class of antidepressants. Here, an explanation for this apparent discrepancy is the complexity of central and autonomic 5-HT effects on cardiovascular function, which include bradycardia, associated with activation of $5-\mathrm{HT}_{1 \mathrm{~A}}$ receptors, as well as tachycardia, associated with activation of $5 \mathrm{HT}_{2}$ receptors. It is possible that the effects of SSRIs shift from parasympathetic - as shown hereto sympathetic activation with increasing length of use. Further research is urgently needed on the combination of antidepressants and physical activity over longer-time frames than typical clinical trials (i.e., years rather than months).

A number of limitations are worth noting. Participants correctly guessed treatment condition beyond chance due to side effects, consistent with those typically experienced with escitalopram (Cipriani et al., 2009b). However, neither experience of side effects, nor accuracy of treatment guess impacted on HR or HRV. Experimenters were also blinded to individual participants' physical activity levels and participants were not aware that physical activity was of primary interest. It is therefore unlikely that the experience of side effects and correctly guessing treatment would have confounded the obtained findings. Another limitation is the use of a questionnaire based-measure of physical activity. It is possible that our measure reflects recent regular physical activity, rather than fitness per se. Future studies could consider use of objective measures of fitness such as $\mathrm{VO}_{2}$ max to confirm that those engaging in regular activity are indeed characterized by higher levels of fitness. Future studies with clinical samples and chronic treatment are also needed to further examine the utility of combining antidepressant medication and vigorous physical activity. 
In summary, results from this experimental study highlight the adverse impact of stress on vagally mediated cardiovascular function and the beneficial moderating effects of escitalopram as well as regular vigorous physical exercise. Escitalopram was observed to increase vagal function under both rest and stress conditions, and under stress, these effects are specific to irregular exercisers, but not regular exercisers. We propose a simplified model for understanding the beneficial effects of physical activity and antidepressants on cardiovascular function that may subsequently lead to improved mental and physical activity. Our study and model provide a foundation on which future research could be based. Increasing concerns about the bidirectional relationship between depression and CVD, reinforce the need for further research on the impact of antidepressants, physical activity and their combination, that is not only focused on amelioration of depressive symptoms, but also on vagally mediated cardiovascular function.

\section{DISCLOSURE}

This research was supported by an Australian Research Council Discovery Project Grant (DP0987332), a National

\section{REFERENCES}

Åberg, M. A. I., Nyberg, J., Torén, K., Sörberg, A., Kuhn, H. G., and Waern, M. (2013). Cardiovascular fitness in early adulthood and future suicidal behaviour in men followed for up to 42 years. Psychol. Med. 1-10. doi: 10.1017/S00332917 13001207

Arunrut, T., Alejandre, H., Chen, M., Cha, J., and Russo-Neustadt, A. (2009). Differential behavioral and neurochemical effects of exercise, reboxetine and citalopram with the forced swim test. Life Sci. 84, 584-589. doi: 10.1016/j.lfs.2009. 02.005

Babyak, M., Blumenthal, J. A., Herman, S., Khatri, P., Doraiswamy, M., Moore, K., et al. (2000). Exercise treatment for major depression: maintenance of therapeutic benefit at 10 months. Psychosom. Med. 62, 633-638.

Blumenthal, J. A., Sherwood, A., Babyak, M. A., Watkins, L. L., Smith, P. J., Hoffman, B. M., et al. (2012). Exercise and pharmacological treatment of depressive symptoms in patients with coronary heart disease: results from the UPBEAT (Understanding the Prognostic Benefits of Exercise and Antidepressant Therapy) study. J. Am. Coll. Cardiol. 60, 1053-1063. doi: 10.1016/j.jacc.2012.04.040

Boullosa, D. A., Abreu, L., Tuimil, J. L., and Leicht, A. S. (2012). Impact of a soccer match on the cardiac autonomic control of referees. Eur. J. Appl. Physiol. 112, 2233-2242. doi: 10.1007/s00421-011-2202-y
Cipriani, A., Furukawa, T. A., Salanti, G., Geddes, J. R., Higgins, J. P., Churchill, R., et al. (2009a). Comparative efficacy and acceptability of 12 new-generation antidepressants: a multipletreatments meta-analysis. Lancet 373, 746-758. doi: 10.1016/S01406736(09)60046-5

Cipriani, A., Santilli, C., Furukawa, T. A., Signoretti, A., Nakagawa, A., McGuire, H., et al. (2009b). Escitalopram versus other antidepressive agents for depression. Cochrane Database Syst. Rev. CD006532. doi: 10.1002/14651858. CD006532.pub2

Cohen, J. (1988a). Set correlation and contingency tables. Appl. Psych. Meas. 12, 425-434. doi: 10.1177/ 014662168801200410

Cohen, J. (1988b). Statistical Power Analysis for the Behavioral Sciencies. Hillsdale, New Jersey: Lawrence Erlbaum Associates.

Cohen, S., Janicki-Deverts, D., and Miller, G. E. (2007). Psychological stress and disease. JAMA 298, 1685-1687. doi: 10.1001/jama.298. 14.1685

Craig, C. L., Marshall, A. L., Sjöström, M. M., Bauman, A. E., Booth, M. L., Ainsworth, B. E., et al. (2003). International physical activity questionnaire: 12 -country reliability and validity. Medicine and science in sports and exercise 35, 1381-1395. doi: $10.1249 / 01 . M S S .0000078924$. 61453.FB Cotman, C. W. (2007). Wheel running and fluoxetine antidepressant
Engesser-Cesar, C., Anderson, A. J., and

Health and Medical Research Council (NHMRC) Project Grant (464863) and a NHMRC Career Development Award (571101) awarded to Andrew H. Kemp. The authors Andrew H. Kemp and Tim Outhred are currently supported by an International Visiting Research Professorship from the Universidade de São Paulo and an Australian Postgraduate Award, respectively. Camilla S Hanson was supported by a Charles Perkins Centre Summer Research Scholarship.

\section{ACKNOWLEDGMENTS}

This research was supported by an Australian Research Council Discovery Project Grant (DP0987332) and a National Health and Medical Research Council Project Grant (464863) awarded to Andrew H. Kemp. The authors would like to acknowledge support and contributions of the following people at different stages of the project: Prof Richard Bryant, Dr Kim Felmingham, Kristi Griffiths, Sasha Saunders, Kristy-Lee Feilds, Mimi Leith, Daniel Quintana, James Heathers, Caroline Fields, Jonathan Kreiger, Sara Shahrestani, Julie Ji, and Matthew Beauregard.

treatment have differential effects in the hippocampus and the spinal cord. Neuroscience 144, 1033-1044. doi: $10.1016 /$ j.neuroscience. 2006 . 10.016

Fernandez, A., Bang, S. E., Srivathsan, K., and Vieweg, W. V. R. (2007). Cardiovascular side effects of newer antidepressants. Anadolu Kardiyol Derg 7, 305-309.

Follesa, P., Biggio, F., Gorini, G., Caria, S., Talani, G., Dazzi, L., et al. (2007). Vagus nerve stimulation increases norepinephrine concentration and the gene expression of BDNF and bFGF in the rat brain. Brain Res. 1179, 28-34. doi: 10.1016/j.brainres. 2007.08.045

Gamelin, F. X., Berthoin, S., and Bosquet, L. (2006). Validity of the polar S810 heart rate monitor to measure R-R intervals at rest. Med. Sci. Sports Exerc. 38, 887-893. doi: $10.1249 / 01 . m s s .0000218135$. $79476.9 \mathrm{c}$

Gandara, B. K., Leresche, L., and Mancl, L. (2007). Patterns of salivary estradiol and progesterone across the menstrual cycle. Ann. N.Y Acad. Sci. 1098, 446-450. doi: 10.1196/annals.1384.022

Geisler, F. C. M., Kubiak, T., Siewert, K., and Weber, H. (2013). Cardiac vagal tone is associated with social engagement and selfregulation. Biol. Psychol. 93, 279-286. doi: 10.1016/j.biopsycho. 2013.02.013

Geisler, F. C. M., Vennewald, N., Kubiak, T., and Weber, H. (2010). The impact of heart rate variability on subjective well-being is mediated by emotion regulation. Pers. Individ. Dif. 49, 723-728. doi: 10.1016/j.paid.2010.06.015

Golding, M., Kotlyar, M., Carson, S. W., Hoyler, S., Lazarus, C., Davidson, C., et al. (2005). Effects of paroxetine on cardiovascular response to mental stress in subjects with a history of coronary artery disease and no psychiatric diagnoses. Psychopharmacology 182, 321-326. doi: 10.1007/s00213-005-0075-7

Hamer, M., and Stamatakis, E. (2009). Physical activity and risk of cardiovascular disease events: inflammatory and metabolic mechanisms. Med. Sci. Sports Exerc. 41, 1206-1211. doi: 10.1249/MSS.0b013e3181971247

Hamer, M., Stamatakis, E., and Steptoe, A. (2009). Dose-response relationship between physical activity and mental health: the Scottish Health Survey. Br. J. Sports Med. 43, 1111-1114. doi: 10.1136/bjsm.2008.046243

Hamer, M., and Steptoe, A. (2007). Association between physical fitness, parasympathetic control, and proinflammatory responses to mental stress. Psychosom. Med. 69, 660-666. doi: 10.1097/PSY.0b013e318148c4c0

Harmer, C. J. (2010). Antidepressant drug action: a neuropsychological perspective. Depress. Anxiety 27, 231-233. doi: 10.1002/da.20680

Harmer, C. J., Goodwin, G. M., and Cowen, P. J. (2009). Why do antidepressants take so long to work. A cognitive neuropsychological model 
of antidepressant drug action. $\mathrm{Br}$. J. Psychiatry 195, 102-108. doi: 10.1192/bjp.bp.108.051193

Hjortskov, N., Rissén, D., Blangsted, A. K., Fallentin, N., Lundberg, U., and Søgaard, K. (2004). The effect of mental stress on heart rate variability and blood pressure during computer work. Eur. J. Appl. Physiol. 92, 84-89. doi: 10.1007/s00421-004-1055-z

Huston, J. M., and Tracey, K. J. (2010). The pulse of inflammation: heart rate variability, the cholinergic antiinflammatory pathway and implications for therapy. J. Intern. Med. 269, 45-53. doi: 10.1111/j.13652796.2010.02321.x

Hynynen, E., Uusitalo, A., Konttinen, N., and Rusko, H. (2008). Cardiac autonomic responses to standing up and cognitive task in overtrained athletes. Int. J. Sports Med. 29, 552-558. doi: 10.1055/s-2007989286

Jiang, W., Velazquez, E. J., Kuchibhatla, M., Samad, Z., Boyle, S. H., Kuhn, C., et al. (2013). Effect of escitalopram on mental stressinduced myocardial ischemiaresults of the REMIT trialescitalopram and stress-induced myocardial ischemia. JAMA 309, 2139-2149. doi: 10.1001/jama.2013.5566

Jönsson, P., Wallergård, M., Österberg, K., Hansen, A. M., Johansson, G., and Karlson, B. (2010). Cardiovascular and cortisol reactivity and habituation to a virtual reality version of the trier social stress test: a pilot study. Psychoneuroendocrinology 35, 1397-1403. doi: 10.1016/j. psyneuen.2010.04.003

Kashdan, T. B., and Rottenberg, J. (2010). Psychological flexibility as a fundamental aspect of health. Clin. Psychol. Rev. 30, 865-878. doi: 10.1016/j.cpr.2010.03.001

Kasper, S., Sacher, J., Klein, N., Mossaheb, N., Attarbaschi-Steiner, T., Lanzenberger, R., et al. (2009). Differences in the dynamics of serotonin reuptake transporter occupancy may explain superior clinical efficacy of escitalopram versus citalopram. Int. Clin. Psychopharmacol. 24, 119-125. doi: 10.1097/YIC.0b013e32832a8ec8

Kemp, A., Gray, M., Silberstein, R., Armstrong, S., and Nathan, P. (2004). Augmentation of serotonin enhances pleasant and suppresses unpleasant cortical electrophysiological responses to visual emotional stimuli in humans. Neuroimage 22, 1084-1096. doi: $\quad 10.1016 /$ j.neuroimage.2004. 03.022
Kemp, A. H., Gordon, E., Rush, A., and Willams, L. M. (2008) Improving the prediction of treatment response in depression: integration of clinical, cognitive, nsychophysiological, neuroimaging, and genetic measures. CNS Spectr. 13, 1066-1086.

Kemp, A. H., and Nathan, P. J. (2004) Acute augmentation of serotonin suppresses cardiovascular responses to emotional valence. Int. J. Neuropsychopharmacol. 7, 65-70. doi: 10.1017/S1461145703003894

Kemp, A. H., and Quintana, D. S. (2013). The relationship between mental and physical health: insights from the study of heart rate variability. Int. J. Psychophysiol. doi: 10. 1016/j.ijpsycho.2013.06.018. [Epub ahead of print].

Kemp, A. H., Quintana, D. S., Gray, M. A., Felmingham, K. L., Brown K., and Gatt, J. M. (2010). Impact of depression and antidepressant treatment on heart rate variability: a review and meta-analysis. Biol. Psychiatry 67, 1067-1074. doi: 10.1016/j.biopsych.2009.12.012

Kessler, R. (2003). Epidemiology of women and depression. J. Affect. Disord. 74, 5-13. doi: 10.1016/S0165-0327(02)00426-3

Khan, A., Brodhead, A. E., Schwartz, K. A., Kolts, R. L., and Brown, W. A. (2005). Sex differences in antidepressant response in recent antidepressant clinical trials. J. Clin. Psychopharmacol. 25, 318-324. doi: $\quad 10.1097 / 01 . j c p .0000168879$. 03169.ce

Kirschbaum, C., Pirke, K. M., and Hellhammer, D. H. (1993). The “Trier Social Stress Test-" a tool for investigating psychobiological stress responses in a laboratory setting. Neuropsychobiology 28, 76-81. doi: 10.1159/000119004

Kleiger, R., Stein, P., and Bigger, J. Jr. (2005). Heart rate variability: measurement and clinical utility. Ann. Noninvasive Electrocardiol. 10, 88-101. doi: 10.1111/j.1542-474X. 2005.10101.x

Kok, B. E., and Fredrickson, B. L. (2010). Upward spirals of the heart: autonomic flexibility, as indexed by vagal tone, reciprocally and prospectively predicts positive emotions and social connectedness. Biol. Psychol. 85, 432-436. doi: 10.1016/j.biopsycho. 2010.09.005

Kroenke, K., Spitzer, R. L., and Williams, J. B. (2001). The PHQ-9: validity of a brief depression severity measure. J. Gen. Intern. Med. 16, 606-613. doi: 10.1046/j.15251497.2001.016009606.x
Langan, J., Mercer, S. W., and Smith, D. J. (2013). Multimorbidity and mental health: can psychiatry rise to the challenge. $\mathrm{Br}$. J. Psychiatry 202, 391-393. doi: 10.1192/bjp.bp.112.123943

Leitzmann, M. F., Park, Y., Blair, A., Ballard-Barbash, R., Mouw, T. Hollenbeck, A. R., et al. (2007). Physical activity recommendations and decreased risk of mortality. Arch. Intern. Med. 167, 2453-2460. doi: 10.1001/archinte.167.22.2453

Lemogne, C., Thomas, F., Consoli, S. M., Pannier, B., Jégo, B., and Danchin, N. (2011). Heart rate and completed suicide: evidence from the IPC cohort study. Psychosom. Med. 73, 731-736. doi: 10.1097/PSY.0b013e3182365dc7

Li, Z., Snieder, H., Su, S., Ding, X. Thayer, J. F., Treiber, F. A., et al. (2009). A longitudinal study in youth of heart rate variability at rest and in response to stress. Int J. Psychophysiol. 73, 212-217. doi: 10.1016/j.ijpsycho.2009.03.002

Licht, C. M. M., de Geus, E. J. C. van Dyck, R., and Penninx, B. W. J. H. (2010a). Longitudinal evidence for unfavorable effects of antidepressants on heart rate variability. Biol. Psychiatry 68 861-868. doi: 10.1016/j.biopsych. 2010.06.032

Licht, C. M. M., Geus, E. J. C., Dyck, R., and Penninx, B. W. J. H. (2010b) Longitudinal evidence for unfavorable effects of antidepressants on heart rate variability. Biol. Psychiatry 68, 1-8. doi: 10.1016/j.biopsych 2010.06.032

Licht, C. M. M., de Geus, E. J. C. Zitman, F. G., Hoogendijk, W. J. G., van Dyck, R., and Penninx, B. W. J. H. (2008). Association between major depressive disorder and heart rate variability in the Netherlands Study of Depression and Anxiety (NESDA). Arch. Gen. Psychiatry 65, 1358-1367. doi: 10.1001/archpsyc.65.12.1358

Lu, Y., Bentley, G. R., Gann, P. H., Hodges, K. R., and Chatterton, R. T. (1999). Salivary estradiol and progesterone levels in conception and nonconception cycles in women: evaluation of a new assay for salivary estradiol. Fertil. Steril. 71, 863-868. doi: 10.1016/S0015-0282 (99)00093-X

Madden, K., and Savard, G. K. (1995) Effects of mental state on heart rate and blood pressure variability in men and women. Clin. Physiol. 15, 557-569. doi: 10.1111/j.1475097X.1995.tb00544.x

Malhi, G. S. (2012). Bipolar antidepressant agents: shaken not stirred. Aust.
N. Z. J. Psychiatry. 46, 289-292. doi: $10.1177 / 0004867412445131$

Mathers, C., and Loncar, D. (2006). Projections of global mortality and burden of disease from 2002 to 2030. PLoS Med. 3:e442. doi: 10.1371/ journal.pmed.0030442

Mills, E. J., Chan, A.-W., Wu, P., Vail, A., Guyatt, G. H., and Altman, D. G. (2009). Design, analysis, and presentation of crossover trials. Trials 10, 27. doi: 10.1186/ 1745-6215-10-27

Mora, S., Cook, N., Buring, J. E., Ridker, P. M., and Lee, I.-M. (2007). Physical activity and reduced risk of cardiovascular events. Circulation 116, 2110-2118. doi: 10.1161/ CIRCULATIONAHA.107.729939

Nemeroff, C. B., and GoldschmidtClermont, P. J. (2012). Heartache and heartbreak-the link between depression and cardiovascular disease. Nat. Publishing Group 9, 526-539. doi: 10.1038/nrcardio.2012.91

Nolen-Hoeksema, S. (2001). Gender differences in depression. Curr. Dir. Psychol. Sci. 10, 173-176. doi: 10.1111/1467-8721.00142

Oveis, C., Cohen, A. B., Gruber, J., Shiota, M. N., Haidt, J., and Keltner, D. (2009). Resting respiratory sinus arrhythmia is associated with tonic positive emotionality. Emotion 9, 265-270. doi: 10.1037/ a0015383

Pavlov, V. A., and Tracey, K. J. (2012). The vagus nerve and the inflammatory reflex-linking immunity and metabolism. Nat. Rev. Endocrinol. 8, 743-754. doi: 10.1038/nrendo.2012.189

Porges, S. W. (1995). Cardiac vagal tone: a physiological index of stress. Neurosci. Biobehav. Rev. 19, 225-233. doi: 10.1016/0149-7634 (94)00066-A

Powell, K. E., Paluch, A. E., and Blair, S. N. (2011). Physical activity for health: What kind. How much? How intense? On top of what? Annu. Rev. Public Health 32, 349-365. doi: 10.1146/annurevpublhealth-031210-101151

Quintana, D. S., Heathers, J. A. J., and Kemp, A. H. (2012). On the validity of using the Polar RS800 heart rate monitor for heart rate variability research. Eur. J. Appl. Physiol. 112, 4179-4180. doi: 10.1007/s00421012-2453-2

Rajendra Acharya, U., Paul Joseph, K., Kannathal, N., Lim, C. M. and Suri, J. S. (2006). Heart rate variability: a review. Med. Biol. Eng. Comput. 44, 1031-1051. doi: 10.1007/s11517-006-0119-0 
Rao, N. (2007). The clinical pharmacokinetics of escitalopram. Clin. Pharmacokinet. 46, 281-290. doi: $\quad 10.2165 / 00003088-2007460$ 40-00002

Rennie, K. L. (2003). Effects of moderate and vigorous physical activity on heart rate variability in a british study of civil servants. Am. J. Epidemiol. 158, 135-143. doi: 10.1093/aje/kwg120

Rudisch, B., and Nemeroff, C. B. (2003). Epidemiology of comorbid coronary artery disease and depression. Biol. Psychiatry 54, 227-240. doi: 10.1016/S0006-3223 (03)00587-0

Russ, T. C., Stamatakis, E., Hamer, M., Starr, J. M., Kivimaki, M., and Batty, G. D. (2012). Association between psychological distress and mortality: individual participant pooled analysis of 10 prospective cohort studies. BMJ 345:e4933. doi: 10.1136/bmj.e4933

Senn, S. (1994). The AB/BA crossover: past, present and future. Stat. Methods Med. Res. 3, 303-324. doi: 10.1177/096228029400300402

Senn, S., D'Angelo, G., and Potvin, D. (2004). Carry-over in cross-over trials in bioequivalence: theoretical concerns and empirical evidence. Pharm. Stat. 3, 133-142. doi: 10.1002/pst.111

Slade, T., Johnston, A., Oakley Browne, M. A., Andrews, G., and Whiteford, H. (2009). 2007 National survey of mental health and wellbeing: methods and key findings. Aus. N.Z. J. Psychiatry 43, 594-605. doi: 10.1080/000486709 02970882

Sogaard, B. (2005). The pharmacokinetics of escitalopram after oral and intravenous administration of single and multiple doses to healthy subjects. J. Clin. Pharmacol. 45, 1400-1406. doi: $10.1177 / 0091270005280860$
Sondermeijer, H. P., van Marle, A. G. J., Kamen, P., and Krum, H. (2002). Acute effects of caffeine on heart rate variability. Am. J. Cardiol. 90, 906-907. doi: 10.1016/S00029149(02)02725-X

Spitzer, R. L., Kroenke, K., Williams, J. B. W., and Löwe, B. (2006). A brief measure for assessing generalized anxiety disorder: the GAD-7. Arch. Intern. Med. 166, 1092-1097. doi: 10.1001/archinte.166.10.1092

Steptoe, A., and Kivimäki, M. (2012). Stress and cardiovascular disease. Nat. Rev. Cardiol. 9, 360-370. doi: 10.1038/nrcardio. 2012.45

Straneva-Meuse, P. (2004a). Bupropion and paroxetine differentially influence cardiovascular and neuroendocrine responses to stress in depressed patients. J. Affect. Disord. 79, 51-61. doi: 10.1016/S0165-0327 (02)00352-X

Straneva-Meuse, P. (2004b). Bupropion and paroxetine differentially influence cardiovascular and neuroendocrine responses to stress in depressed patients. J. Affect. Disord. 79, 51-61. doi: 10.1016/S01650327(02)00352-X

Tabachnick, B. G., and Fidell, L. S. (2007). Using multivariate statistics 5th edn. Boston: Pearson Education, Inc.

Task Force of the European Society of Cardiology, and the North American Society of Pacing and Electrophysiology. (1996). Heart rate variability. standards of measurement, physiological interpretation, and clinical use. Eur. Heart J. 17, 354-381. doi: 10.1093/oxfordjournals.eurheartj.a014868

Thayer, J. F., and Brosschot, J. F. (2005). Psychosomatics and psychopathology: looking up and down from the brain. Psychoneuroendocrinology 30, 1050-1058. doi: 10.1016/j. psyneuen.2005.04.014
Thayer, J. F., Hansen, A. L., SausRose, E., and Johnsen, B. H. (2009). Heart rate variability, prefrontal neural function, and cognitive performance: the neurovisceral integration perspective on self-regulation, adaptation, and health. Ann. Behav. Med. 37, 141-153. doi: 10.1007/s12160-0099101-z

Thayer, J. F., and Sternberg, E. (2006) Beyond heart rate variability: vagal regulation of allostatic systems. Ann. N.Y. Acad. Sci. 1088, 361-372. doi 10.1196/annals.1366.014

Thayer, J. F., Yamamoto, S. S., and Brosschot, J. F. (2010a). The relationship of autonomic imbalance, heart rate variability and cardiovascular disease risk factors. Int. J. Cardiol. 141, 122-131. doi: 10.1016/j.ijcard.2009.09.543

Thayer, J. F., Yamamoto, S. S., and Brosschot, J. F. (2010b). The relationship of autonomic imbalance, heart rate variability and cardiovascular disease risk factors. Int. J. Cardiol. 141, 122-131. doi: 10.1016/j.ijcard.2009.09.543

Tracey, K. (2002). The inflammatory reflex. Nature 420, 853-859. doi: 10.1038/nature01321

Vermetten, E. (2006). Alterations in stress reactivity after longterm treatment with paroxetine in women with posttraumatic stress disorder. Ann. N.Y. Acad. Sci. 1071, 184-202. doi: 10.1196/annals.1364.014

Weippert, M., Kumar, M., Kreuzfeld, S., Arndt, D., Rieger, A., and Stoll, R. (2010). Comparison of three mobile devices for measuring $\mathrm{R}-\mathrm{R}$ intervals and heart rate variability: Polar S810i, Suunto t6 and an ambulatory ECG system. Eur. J. Appl. Physiol. 109, 779-786. doi: 10.1007/s00421010-1415-9

Whang, W., Kubzansky, L. D., Kawachi, I., Rexrode, K. M., Kroenke, C.
H., Glynn, R. J., et al. (2009). Depression and risk of sudden cardiac death and coronary heart disease in women. J. Am. Coll. Cardiol. 53, 950-958. doi: 10.1016/j.jacc.2008.10.060

Conflict of Interest Statement: Gin S. Malhi has received research support from AstraZeneca, Eli Lilly, Organon, Pfizer, Servier, and Wyeth. He has been a speaker for AstraZeneca, Eli Lilly, Janssen Cilag, Lundbeck, Pfizer, Ranbaxy, Servier, and Wyeth. He has been a consultant for AstraZeneca, Eli Lilly, Janssen Cilag, Lundbeck, and Servier. Andre R. Brunoni declares no potential conflict of interest.

Received: 24 June 2013; accepted: 03 September 2013; published online: 24 September 2013.

Citation: Hanson CS, Outhred T, Brunoni AR, Malhi GS and Kemp AH (2013) The impact of escitalopram on vagally mediated cardiovascular function to stress and the moderating effects of vigorous physical activity: a randomized controlled treatment study in healthy participants. Front. Physiol. 4:259. doi: 10.3389/fphys.2013.00259

This article was submitted to Clinical and Translational Physiology, a section of the journal Frontiers in Physiology.

Copyright (c) 2013 Hanson, Outhred, Brunoni, Malhi and Kemp. This is an open-access article distributed under the terms of the Creative Commons Attribution License (CC BY). The use, distribution or reproduction in other forums is permitted, provided the original author(s) or licensor are credited and that the original publication in this journal is cited, in accordance with accepted academic practice. No use, distribution or reproduction is permitted which does not comply with these terms. 